\title{
Comparison of TaqMan ${ }^{\circledR}$ Assay and PCR- sequencing Method for Analyzing CYP2D10*6 and CYP2D4*6 Alleles: a False Negative Issue
}

\author{
Zahra Khalaj ${ }^{1,2}$, Zohreh Baratieh ${ }^{1}$, Parvaneh Nikpour ${ }^{1,3}$, Fariborz \\ Mokarian ${ }^{4}$, Hossein Khanahmad ${ }^{1}$, Rasoul Salehi ${ }^{1,3}$, Mansoor \\ Salehi ${ }^{1,2,3, *}$ \\ 1 Department of Genetics and Molecular Biology, Faculty of Medicine, Isfahan \\ University of Medical Sciences, Isfahan, Iran \\ ${ }^{2}$ Medical Genetic Center of Genome, Isfahan, Iran \\ ${ }^{3}$ Child Growth and Development Research Center, Research Institute for Primordial \\ Prevention of Non-communicable Disease, Isfahan University of Medical Sciences, \\ Isfahan, Iran \\ ${ }^{4}$ Cancer Prevention Research Center, Isfahan University of Medical Sciences, Isfahan, \\ Iran \\ ${ }^{*}$ Corresponding author: Mansoor Salehi, Department of Genetics and Molecular \\ Biology, Faculty of Medicine, Isfahan University of Medical Sciences, Isfahan, Iran. \\ E-mail:m_salehi@med.mui.ac.ir
}

DOI: $10.21859 / \mathrm{mci}-$ supp-22

\section{Keywords:}

CYP2D6*4

CYP2D6*10

Genotyping

TaqMan $^{\circledast}$

PCR-sequencing

Pseudogenes

\begin{abstract}
Introduction: Pharmacogenetics is the study of inherited genetic variations in pathways responsible for metabolism of drugs and their effects on the individual responses. Cytochrome P450 2D6 (CYP2D6) is an important drug metabolizing enzyme involved in the metabolism of many drugs. The CYP2D6 gene is highly polymorphic and currently more than 100 allelic variants have been reported for it. The combination of different alleles yields different phenotypes such as: extensive metabolizer (EM), intermediate metabolizer (IM) and poor metabolizer (PM). The most important IM allele with reduced activity of the enzyme is CYP2D6*10. Variant CYP2D6*4 is the most important null allelic variant responsible for eliminating enzymatic activity and a poor metabolism (PM). Genotyping of these two alleles in the population for assessing the efficacy of drug is of particular importance. TaqMan ${ }^{\circledast}$ assays are widely utilized for genotyping CYP2D6 alleles, but due to the existence of two pseudogenes, CYP2D7 and CYP2D8, and highly polymorphic nature of the gene, assay design can be challenging.

Materials and Methods: Here we compared TaqMan ${ }^{\circledast}$ allelic discrimination assay and PCR-sequencing method for genotyping CYP2D6* 10 and CYP2D6*4 in 134 breast cancer patients in Isfahan province of Iran.

Results: According to our study, the results of both methods for CYP2D6*4 allele were concordant with the reported allele frequency of $10.4 \%$. However, as the results of the two methods for genotyping CYP2D6* 10 allele were contradictory, we conducted more experiments to examine the reasons behind these conflicting results.

Conclusions: Taken together, our findings illustrate that TaqMan ${ }^{\circledast}$ assay may be not precise enough to genotype all CYP2D6 alleles; therefor other alternative genotyping methods should be considered.
\end{abstract}

